\title{
Increase of Reactive Power Compensation Efficiency on the Basis of Individual Phase Compensation Devices
}

\author{
Sergey V. Kuzmin, Artem A. Zavalov*, \\ Roman S. Kuzmin and Vitalii A. Menshikov \\ Siberian Federal University \\ Krasnoyarsk, Russian Federation
}

Received 13.05.2019, received in revised form 03.07.2019, accepted 12.10.2019

Abstract. The purpose of this article is to establish an effective method for compensating reactive power in four-wire networks voltage up to $1000 \mathrm{~V}$ with a solidly grounded neutral. The method and the device for independent individual phase reactive power compensation are presented. The known devices and methods for symmetric reactive power compensation in three-phase four-wire networks are ineffective. Under certain circumstances, probability of emergency mode increases. Asymmetrical and unbalanced load on network phases dominate in three-phase four-wire networks. As a result, current in the neutral wire increases several times. This can lead to thermal destruction of the neutral wire and failure of power supply cable. By reducing load unbalance in network phases and eliminating effects of undercompensation and overcompensation, the method of independent individual phase reactive power compensation makes it possible to reduce the current in the neutral. The method of independent individual phase compensation is preferred when using devices reactive power compensation in three-phase networks with solidly grounded neutral. This method allows to increase transfer capacity, to reduce losses, and also reduces chance of emergency mode. Results of introduction of devices for individual phase reactive power compensation into operation prove the efficiency of the proposed method.

Keywords: reactive power, power factor, nonsymmetrical load, unbalanced load, individual phase compensation.

Citation: Kuzmin S.V., Zavalov A.A., Kuzmin R.S., Menshikov V.A. Increase of reactive power compensation efficiency on the basis of individual phase compensation devices, J. Sib. Fed. Univ. Eng. \& Technol., 2020, 13(1), 14-24. DOI: 10.17516/1999494X-0187

(C) Siberian Federal University. All rights reserved

This work is licensed under a Creative Commons Attribution-NonCommercial 4.0 International License (CC BY-NC 4.0).

* Corresponding author E-mail address: zavalovartem@mail.ru 


\title{
Повышение эффективности компенсации
}

\section{реактивной мощности}

\section{на основе устройств пофазной компенсации}

\author{
С.В. Кузьмин, А.А. Завалов, \\ P.С. Кузьмин, В.А. Меньшиков \\ Сибирский федеральный университет \\ Российская Федераиия, Красноярск
}

\begin{abstract}
Аннотация. Целью данной статьи является установление эффективного способа компенсации реактивной мощности в четырехпроводных сетях с глухозаземленной нейтралью общего назначения напряжением до 1000 В. Представлен метод и устройство пофазной независимой компенсации реактивной мощности. Известные устройства и методики симметричной компенсации реактивной мощности в трехфазных четырехпроводных сетях оказываются неэффективны. При определенных обстоятельствах возрастает вероятность возникновения аварийного режима вследствие того, что в трехфазных четырехпроводных сетях преобладает несимметричная и/или неравномерная нагрузка по фазам сети, ток в нейтральном проводе возрастает в разы. Это может привести к термическому разрушению нулевого провода и выходу из строя силового кабеля. Метод пофазной независимой компенсации реактивной мощности позволяет уменьшить ток в нейтрали за счет уменьшения несимметрии нагрузки в фазах сети и исключения эффектов недокомпенсации и перекомпенсации. Метод пофазной независимой компенсации является более предпочтительным при использовании средств компенсации реактивной мощности в трехфазных сетях с глухозаземленной нейтралью и позволяет увеличить пропускную способность, уменьшить потери, а также снижает риск возникновения аварийного режима. Результаты внедрения в эксплуатацию устройств пофазной компенсации реактивной мощности доказывают эффективность предложенного метода.
\end{abstract}

Ключевые слова: реактивная мощность, коэффициент мощности, несимметричная и неравномерная нагрузка, пофазная компенсация.

Цитирование: Кузьмин, С.В. Повышение эффективности компенсации реактивной мощности на основе устройств пофазной компенсации / С.В. Кузьмин, А.А. Завалов, Р.С. Кузьмин, В.А. Меньшиков // Журн. Сиб. федер. ун-та. Техника и технологии, 2020. 13(1). C. 14-24. DOI: 10.17516/1999-494X-0187

Городские сети напряжением до 1000 В эксплуатируются с глухозаземленным режимом нейтрали. Нагрузки городских трехфазных электрических сетей состоят в основном из однофазных электроприемников, подключенных между фазой и нейтральным проводом сети.

Особенностью трехфазных сетей с глухозаземленным режимом нейтрали является наличие разного сдвига фаз между током и напряжением в каждой фазе сети. Значение полной мощности в трехфазных сетях зависит от угла сдвига фаз между током и напряжением. Величина полной мощности однофазной сети определяется нижеследующим выражением:

$$
S=U \cdot I \text {, }
$$

где $S$ - полная мощность, ВА; $U$ - значение напряжения сети, В; $I$ - значение тока сети, А.

Если в сети присутствует только активная составляющая мощности, угол сдвига фаз равен нулю и, соответственно, ток и напряжение совпадают по фазе. В этом случае 


$$
S=P,
$$

где $P$ - активная составляющая мощности, Вт.

Если же в сети помимо активной составляющей мощности также присутствует ее реактивная (индуктивная, емкостная) составляющая, угол сдвига фаз между током и напряжением будет отличным от нуля. Значение полной мощности для трехфазной сети согласно [1] определяется следующим выражением:

$$
S=\sqrt{P^{2}+Q^{2}},
$$

где $Q$ - реактивная составляющая мощности, Вар.

Реактивная составляющая увеличивает значение потребления полной мощности в сети, уменьшает ее пропускную способность, увеличивает потери, а также является бесполезной для потребителя. Некоторая величина реактивной составляющей мощности, присутствующая в сети, незначительна, но при достижении определенных значений требуется ее компенсация.

Реактивная составляющая полной мощности в свою очередь определяется следующим выражением:

$$
Q=U\left(I_{L}-I_{C}\right)=Q_{L}-Q_{C}
$$

где $Q_{L}-$ индуктивная составляющая реактивной мощности, Вар; $Q_{C}$ - емкостная составляющая реактивной мощности, Вар.

Для определения продуктивности работы трехфазной сети переменного тока используют коэффициент мощности. Понятие «коэффициент мощности» ввел Дж. Максвелл в 1864 г. Коэффициент мощности численно равен косинусу угла сдвига фаз и определяется следующим выражением:

$$
\cos \varphi=\frac{P}{S}
$$

Значение коэффициента мощности стремятся свести к единице с помощью специальных устройств и методов компенсации реактивной мощности, тем самым нивелируя негативное воздействие сдвига фаз между током и напряжением. Идея компенсации реактивной составляющей очень стара: первый балансирующий компенсатор был разработан Steinmetz и представлен [2] в 1917 г. Различные устройства и методы были предложены в [3-8].

Для определения уровня потребления реактивной мощности используют коэффициент реактивной мощности, определяющийся следующим выражением:

$$
\operatorname{tg} \varphi=\frac{Q}{P} .
$$

В трехфазных сетях с глухозаземленной нейтралью на значение полной потребляемой мощности помимо реактивной составляющей также оказывают влияние несбалансированные мощности обратной и нулевой последовательностей [9]. В таком случае уравнение полной мощности при синусоидальном напряжении будет иметь следующий вид:

$$
S=\sqrt{P^{2}+\left(Q_{L}^{2}-Q_{C}^{2}\right)+D_{n}^{2}+D_{Z}^{2}},
$$

где $D_{n}$ - несбалансированная составляющая обратной последовательности, Вар; $D_{z}-$ несбалансированная составляющая нулевой последовательности, Вар.

$$
-16-
$$


Появление несбалансированных составляющих мощности обусловлено спецификой данных сетей, заключающейся в том, что в данных сетях распределение нагрузки происходит несимметрично или неравномерно относительно фаз. Вследствие этого по фазам сети начинают протекать токи обратной и нулевой последовательности, что приводит к дальнейшему росту тока в нейтральном проводе и увеличивает риск возникновения перекоса фазных напряжений, уменьшая надежность сетей данного типа.

Необходимость компенсации реактивной составляющей полной мощности в трехфазных сетях с глухозаземленной нейтралью за последние два десятилетия значительно возросла в связи с существенно изменившимися за это время параметрами нагрузки. Так, средневзвешенный коэффициент мощности многоквартирного дома два десятилетия назад не опускался ниже значения 0,95 , тогда как в настоящее время данный показатель может находиться в диапазоне 0,75 - 0,93. Современные жилые дома оснащены большим количеством электроприемников. К ним относятся различные осветительные и бытовые приборы, силовое оборудование. Потребление реактивной мощности коммунально-бытовых потребителей постоянно растет: лампы накаливания заменяют осветительными устройствами с использованием L-C-цепей, все больше теле- и видеоаппаратуры, широко применяются компьютеры, СВЧ-печи, увеличиваются мощности потребителей с использованием электродвигателей: инструмент, стиральные машины, холодильники [10].

При неизменном потреблении активной мощности и растущем потреблении реактивной мощности будет увеличиваться полный ток, из-за чего будут расти потери мощности и падение напряжения в системе электроснабжения. Напряжение на потребителе будет уменьшаться, и если уменьшение будет критическим, т.е. сверх допустимого значения, то нормальная работа вышеупомянутой техники может стать невозможной. Одновременно с увеличением падения напряжения растут потери активной мощности в самих сетях. Установлено, что потери активной мощности в системах электроснабжения, как минимум, обратно пропорциональны квадрату коэффициента мощности. При сопоставлении периодов времени до и после 1995 г. видно, что потери активной мощности в современных системах электроснабжения возросли в 1,56 раза (на $56 \%$ ).

В табл. 1 указаны усредненные коэффициенты мощности и коэффициенты реактивной мощности для различных бытовых приборов, повсеместно используемых в быту.

В табл. 2 приведено количество электроприборов, в среднем используемых в одной квартире многоквартирного жилого дома, для сравнения значений периода до 1995 г. и в настоящее время.

Из анализа данных табл. 1 и 2 видно, что количество электроприемников, используемых в быту, с низким коэффициентом мощности неуклонно растет в последние годы, в связи с этим средневзвешенный коэффициент мощности стремительно уменьшается. Коэффициент мощности есть мера эффективного использования установленной мощности трансформаторов на подстанциях и максимальной пропускной способности электрических сетей. Для повышения коэффициента мощности требуется грамотный подход к компенсации реактивной мощности в городских сетях общего назначения, который должен учитывать следующие факторы:

1) значительный рост использования электроприборов с низким коэффициентом мощности вне объектов и зданий, размещаемых снаружи, в частности на их фасадах, например освещение улиц и рекламы с помощью газоразрядных и светодиодных ламп;

$$
-17-
$$


Таблица 1. Коэффициенты мощности и коэффициенты реактивной мощности бытовых приборов

Table 1. Power factor and reactive power factor of household appliances

\begin{tabular}{|l|c|c|}
\hline \multicolumn{1}{|c|}{ Наименование электроприбора } & $\cos \varphi$ & $\operatorname{tg} \varphi$ \\
\hline Микроволновая печь & 0,75 & 0,882 \\
\hline Телевизор & 0,65 & 1,168 \\
\hline Стиральная машина-автомат & 0,8 & 0,75 \\
\hline Телефон (смартфон) (во время зарядки) & $0.45-0,7$ & $1,98-1,02$ \\
\hline Аккумулятор шуруповерта (во время зарядки) & 0,72 & 1,1 \\
\hline Электродрель & 0,65 & 1,168 \\
\hline Холодильник & 0,7 & 1,02 \\
\hline Ноутбук & 0,65 & 1,168 \\
\hline Фен для волос & 0,7 & 1,02 \\
\hline Вытяжка кухонная & $0,6-0,8$ & $1,33-0,75$ \\
\hline Пылесос & 0,65 & 1,168 \\
\hline Роутер & 0,65 & 1,168 \\
\hline Лампа диодная & $0,3-0,6$ & $3,18-1,33$ \\
\hline Кондиционер & 0,75 & 0,882 \\
\hline Посудомоечная машина & 0,8 & 0,75 \\
\hline Люминесцентные лампы & 0,5 & 1,732 \\
\hline Электроплита & 0,85 & 0,62 \\
\hline Лифт подъемный & 0,65 & 1,168 \\
\hline
\end{tabular}

Таблица 2. Количество электроприборов, используемых в быту до 1995 г. и в период после 2015 г.

Table 2. The number of electrical appliances used in everyday life before 1995 and during the period after 2015

\begin{tabular}{|l|c|c|}
\hline \multicolumn{1}{|c|}{ Наименование электроприбора } & \multicolumn{2}{c|}{ Кол-во приборов } \\
\cline { 2 - 3 } & до 1995 г. & после 2015 г. \\
\hline Микроволновая печь & 0 & 1 \\
\hline Телевизор & 1 & 2 \\
\hline Стиральная машина-автомат & 0 & 1 \\
\hline Телефон (смартфон) & 0 & 3 \\
\hline Шуруповерт & 0 & 1 \\
\hline Электродрель & 0 & 1 \\
\hline Холодильник & 1 & 2 \\
\hline Ноутбук & 0 & 2 \\
\hline Фен для волос & 0 & 1 \\
\hline Вытяжка кухонная & 0 & 1 \\
\hline Пылесос & 1 & 1 \\
\hline Роутер & 0 & 15 \\
\hline Лампа диодная & 0 & 1 \\
\hline Кондиционер & 0 & \\
\hline Посудомоечная машина & 0 & 1 \\
\hline Электроплита & 1 & 1 \\
\hline
\end{tabular}


2) использование лифтов в многоквартирных домах;

3) появление в городах большого количества офисных, административных, торговоразвлекательных зданий, в которых используется оргтехника, диодное освещение, кондиционеры и другие электроприборы с низким коэффициентом мощности.

Проанализируем энергопотребление основных электроприемников муниципальных образований, к которым относятся административно-бытовые здания, учебные заведения, детские сады, торгово-развлекательные комплексы, спортивные сооружения и многоквартирные дома. Для этой цели были выполнены исследования энергопотребления вышеуказанных электроприемников в г. Дудинке. В табл. 3 приведены средневзвешенные значения коэффициента мощности для данных сооружений и домов в зависимости от времени дня и времени года.

Как показал детальный анализ данных табл. 3, значения средневзвешенного коэффициента мощности в исследуемых сетях для всех типов зданий и сооружений заметно меньше в осеннезимний период времени года по сравнению с весенне-летним периодом. Кроме того, можно проследить за тем, что в данных сетях значения средневзвешенного коэффициента мощности для жилых многоквартирных домов, школ и торгово-развлекательных центров уменьшаются в вечернее время суток независимо от времени года. Для детских садов, административных зданий наименьшие значения средневзвешенного коэффициента мощности приходятся на период времени между восьмью и шестнадцатью часами вне зависимости от времени года. Для спортивных сооружений (бассейн) минимальные значения средневзвешенного коэффициента мощности наблюдаются между полуночью и восьмью часами. Для технического лицея наибо-

Таблица 3. Средневзвешенные значения коэффициента мощности в зависимости от времени дня и времени года

Table 3. Weighted average values of power factor depending on time of the day and time of the year

\begin{tabular}{|c|c|c|c|c|c|c|}
\hline \multirow{3}{*}{ Тип здания } & \multicolumn{6}{|c|}{ Коэффициент мощности } \\
\hline & \multicolumn{3}{|c|}{$\begin{array}{c}\text { весенне-летний период } \\
\text { временные интервалы }\end{array}$} & \multicolumn{3}{|c|}{$\begin{array}{l}\text { осенне-зимний период } \\
\text { временные интервалы }\end{array}$} \\
\hline & 0:00-8:00 & $8: 00-16: 00$ & $16: 00-24: 00$ & 0:00-8:00 & $8: 00-16: 00$ & $16: 00-24: 00$ \\
\hline $\begin{array}{l}\text { Средняя общеобразовательная } \\
\text { школа }\end{array}$ & 0,87 & 0,89 & 0,83 & 0,75 & 0,76 & 0,71 \\
\hline Детский сад & 0,92 & 0,81 & 0,87 & 0,85 & 0,68 & 0,77 \\
\hline Технический лицей & 0,96 & 0,89 & 0,93 & 0,92 & 0,83 & 0,81 \\
\hline Торгово-развлекательный центр & 0,93 & 0,94 & 0,91 & 0,78 & 0,78 & 0,78 \\
\hline Бассейн & 0,81 & 0,91 & 0,86 & 0,8 & 0,86 & 0,85 \\
\hline Административное здание & 0,96 & 0,91 & 0,98 & 0,93 & 0,76 & 0,82 \\
\hline $\begin{array}{l}\text { Девятиэтажный } \\
\text { многоквартирный дом }\end{array}$ & 0,92 & 0,93 & 0,88 & 0,83 & 0,87 & 0,75 \\
\hline $\begin{array}{l}\text { Пятиэтажный } \\
\text { многоквартирный дом }\end{array}$ & 0,9 & 0,92 & 0,91 & 0,8 & 0,83 & 0,81 \\
\hline Студенческое общежитие & 0,81 & 0,7 & 0,87 & 0,84 & 0,78 & 0,72 \\
\hline Здание гостиницы & 0,87 & 0,84 & 0,83 & 0,82 & 0,8 & 0,8 \\
\hline
\end{tabular}


лее низкие значения средневзвешенного коэффициента мощности имеют место быть в дневное и вечернее время суток.

Компенсация реактивной мощности осуществляется с помощью специальных компенсирующих устройств, подключенных в определенных точках системы электроснабжения, и с помощью организационных мероприятий по снижению потребления реактивной мощности, которые связаны с естественным уменьшением реактивной мощности [11].

Рассмотрим некоторые компенсирующие устройства, наиболее часто используемые на практике. Эти устройства можно разделить на две группы: вращающиеся синхронные машины и статические компенсаторы. К статическим компенсаторам относятся устройства на базе конденсаторных батарей, которые являются наиболее перспективными для использования в трехфазных сетях напряжением до 1000 В. Впервые конденсаторы для компенсации реактивной мощности установил в 1898 г. П. Бушеро.

Основные преимущества устройств на базе конденсаторных батарей:

небольшие, практически постоянные в зоне номинальной температуры окружающей среды, удельные потери активной мощности конденсаторов, не превышающие 0,5 Вт на 1 квар компенсационной мощности, т.е. не более 0,5 \%;

отсутствие механически перемещаемых частей в процессе эксплуатации;

простота монтажа и эксплуатации;

относительно невысокие капиталовложения;

большой диапазон подбора требуемой мощности;

относительная простота схемы регулирования реактивной мощности;

возможность установки в любых точках электрической сети, бесшумность работы.

В настоящее время в связи с существенно изменяющимся во времени потреблением реактивной мощности используют автоматические конденсаторные установки с симметричной компенсацией по фазам сети. Однако в трехфазных сетях до 1000 В с глухозаземленным режимом нейтрали потребление реактивной мощности для различных фаз значительно отличается. Применение конденсаторных установок с симметричной компенсацией может привести к режимам «перекомпенсации» или «недокомпенсации» реактивной мощности по отдельным фазам сети. Таким образом, для использования в трехфазных сетях с глухозаземленной нейтралью требуется разработка более эффективного метода и устройства компенсации реактивной мощности.

В определенном смысле «фазность» компенсации можно считать еще одним критерием классификации способов компенсации: пофазная противопоставляется симметричной.

Предлагаемый метод независимой пофазной компенсации реактивной мощности заключается в целенаправленной независимой компенсации реактивной мощности в каждой фазе сети. Это позволяет исключить режим «недокомпенсации» или «перекомпенсации» реактивной мощности в отдельных фазах сети и снизить токи в каждой фазе сети. Снижение полных токов в фазах сети уменьшает ток в нейтральном проводе и несбалансированные составляющие полной мощности обратной и нулевой последовательности, повышая пропускную способность сети.

Устройство пофазной независимой компенсации реактивной мощности было успешно внедрено в г. Дудинке. Устройства были установлены на электрических вводах детских садов, 
школ, спортивно-развлекательных комплексов, оздоровительных центров и административных зданий.

Поскольку компенсаторы с соединением в треугольник не могут воздействовать на ток в нейтрали, при пофазной независимой компенсации реактивной мощности конденсаторные батареи собирают по схеме звезда с нулевым проводом. Для защиты конденсаторных батарей от высших гармоник при необходимости используют антирезонансные дроссели, от пусковых токов конденсаторные батареи защищают с помощью контакторов с демпфирующими реакторами.

На основе измерений потребления электрической энергии в школе № 1 г. Дудинки были построены три векторные диаграммы токов, на которых отображены токи до компенсации реактивной мощности, после использования конденсаторных установок с симметричной компенсацией и после использования конденсаторных установок с пофазной независимой компенсацией реактивной мощности (рис. 1).

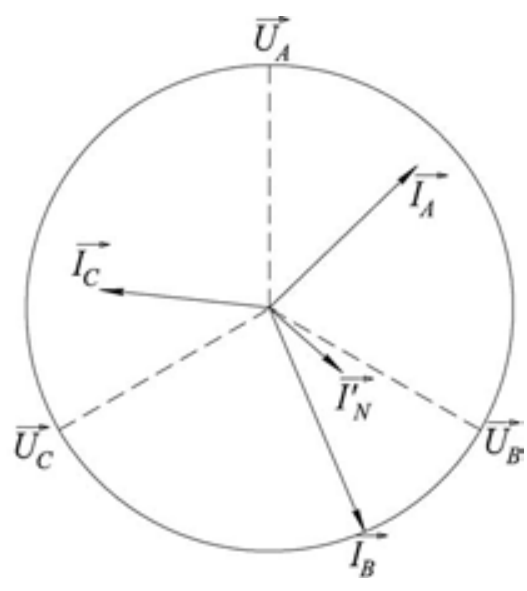

a

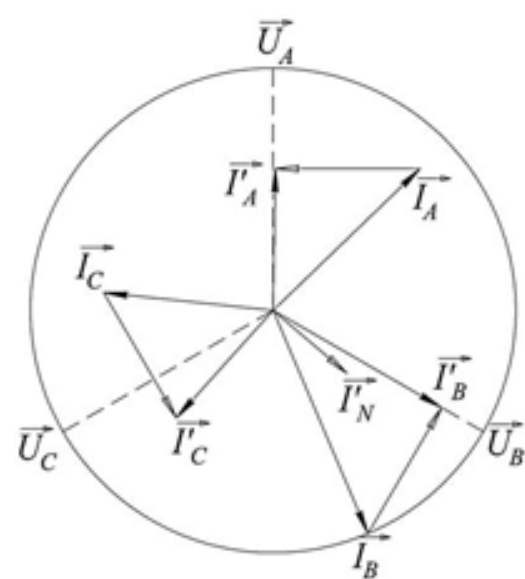

6

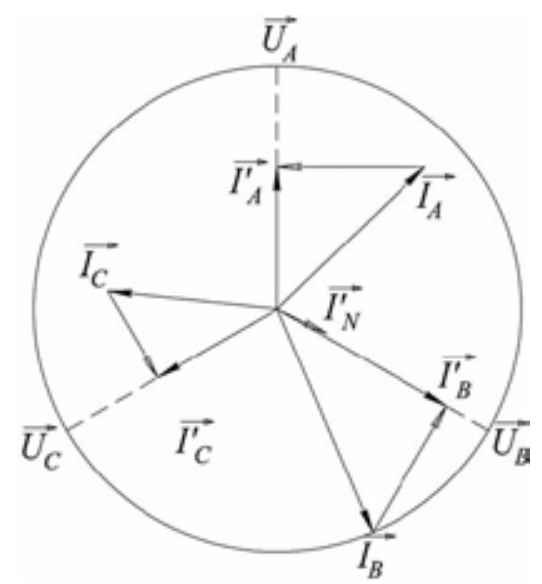

B

Рис. 1. Векторные диаграммы: а - до компенсации; б - компенсация с использованием средств симметричной компенсации; в - компенсация с использованием средств пофазной компенсации

Fig. 1. Vector diagrams: a - before compensation; 6 -symmetrical compensation; в - individual phase compensation 
Векторные диаграммы показывают, что устройство пофазной компенсации реактивной мощности является наиболее эффективным в связи с тем, что коэффициенты мощности в фазах сети близки к единице, токи в фазах сети заметно уменьшились, а ток в нейтральном проводе уменьшился в 2,7 раза. Тогда как после использования устройств с симметричной компенсацией в фазах сети возникают режимы перекомпенсации и недокомпенсации. Ток в нейтральном проводе изменился незначительно, а его величина близка токам в фазах сети.

За счет значительного снижения тока в нейтральном проводе после использования устройств пофазной компенсации реактивной мощности повышается надежность трехфазной сети с глухозаземленной нейтралью. Если нейтральный провод выполнен с меньшим сечением в сравнении с фазными проводами, то значительный ток в нейтральном проводе, близкий по значению токам в фазах сети или превышающий их, в зависимости от длительности максимального режима электропотребления может привести к его термическому разрушению. Разрушение нейтрального провода в свою очередь приведет к перекосу фазных напряжений, в результате которого в одной фазе напряжение может существенно возрасти, а в двух других уменьшиться, что негативно скажется на используемом оборудовании, поскольку оно может выйти из строя из-за повышенного напряжения либо отключиться при уменьшении напряжения. Таким образом, метод и устройство пофазной компенсации реактивной мощности существенно увеличивает надежность использования данного типа сетей.

На рис. 2 представлена гистограмма, построенная на основе измерений потребления электрической энергии в школе № 1 г. Дудинки, на которой можно увидеть, как изменяется ток в фазах сети и нейтральном проводе при использовании пофазной компенсации реактивной мощности.

Уменьшение токов в фазах сети приводит к высвобождению значительной доли потенциальной пропускной способности кабельных линий, которая ранее была занята реактивной

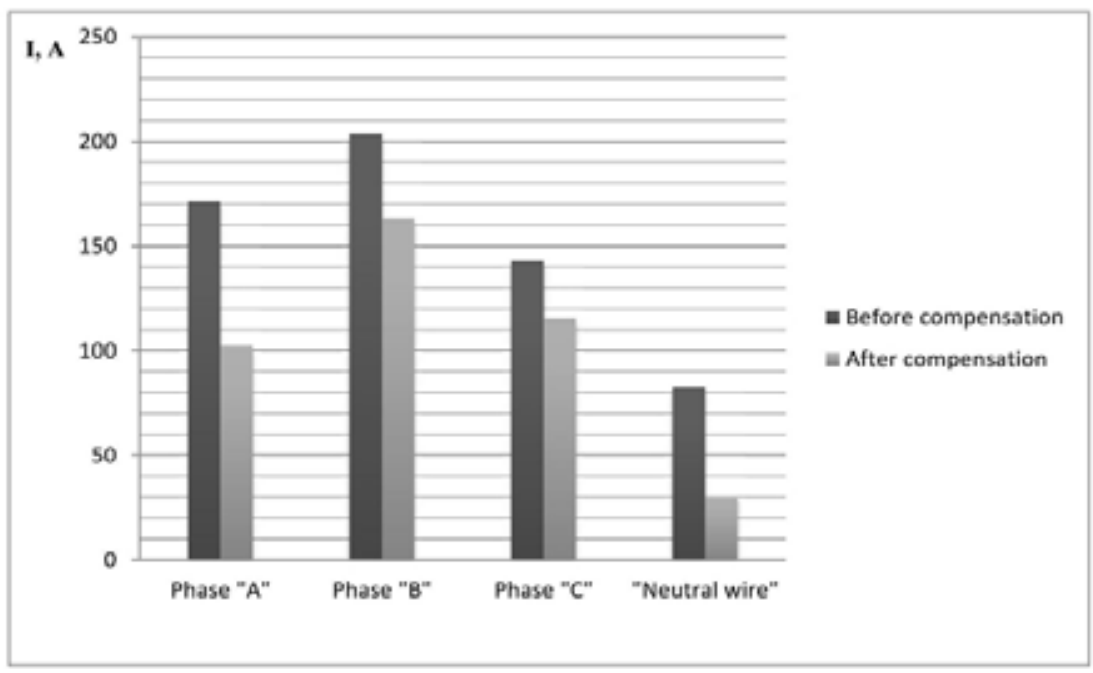

Рис. 2. Гистограмма токов в фазах сети и нейтральном проводе (до и после пофазной компенсации)

Fig. 2. Histogram of currents in phases of network and neutral wire (before compensation and after individual phase compensation) 
составляющей тока, к снижению потерь электроэнергии в сети и поддержанию в рамках нормируемых значений напряжения на потребителе.

\section{Выводы}

1. Для северных территорий выявлена характерная зависимость коэффициента мощности от времени года: в осенне-зимний период года коэффициент мощности весьма мал из-за интенсивного использования современного освещения и бытовых приборов, в весенне-летний период коэффициент мощности возрастает из-за снижения использования бытовых приборов, связанного с отпускным периодом.

2. Использование современных электроприборов и оргтехники приводит к существенному снижению коэффициента мощности.

3. Низкая эффективность симметричной компенсации обусловлена неравномерной нагрузкой по отдельным фазам сети четырехпроводной системы электроснабжения с глухозаземленной нейтралью. Для данного класса сетей наиболее эффективным является устройство пофазной компенсации, что подтверждено положительным опытом эксплуатации в г. Дудинке.

\section{Список литературы / References}

[1] Curtis H.L., Silsbee F.B. Definitions of power and related quantities. Trans. AIEE, 1935, 54, 394-404.

[2] Steinmetz C.P. Theory and Calculation of Electrical Apparatur. New York, USA: McGrawHill, 1917.

[3] Grandpierr M., Trannoy B. Astationary power device to rebalance and compensate reactive power in three-phase network. Proc Ind. Appl. Soc. Annu. Conf., 1977, 127-135.

[4] Czarnecki L.S. Minimization of unbalanced currents in three-phase asymmetrical circuits with nonsinusoidal voltage. Proc. Inst. Elect. Eng., B, 1992, 139(4), 347-354.

[5] Lee S.Y., Wu C.J. On-line reactive power compensation schemes for unbalanced three-phase four wire distribution systems. IEEE Trans. Power Del., 1993, 8(4), 1235-1239.

[6] Oriega L.C., Oliviera De., Barros Neto M.C., de Souza J. B. Load compen-sation in four-wire electrical power systems. Proc. Int. Conf. Power Syst. Technol., 2000, 3, 1975-1580.

[7] Arendse C., Atkinson-Hope G. Design of Steinmetz symmetrizer and application in unbalanced network. UPEC Conf., Cardiff, Wales, U.K., 2010.

[8] Jeon S.J., Willens J.L. Reactive power compensation in multiline systems under sinusoidal unbalanced conditions. Int. J. Circuit Theory Appl., 2011, 39, 211-224.

[9] Czarnecki L.S., Haley P.M. Unbalanced Power in Four-Wire Systems and Its Reactive Compensation, IEEE Trans. Power Del., 2015, 30(1), 53-63.

[10] Можный Е.Г., Степанов А.Г. Проблемы компенсации реактивной мощности. Электроэнергетика и электромеханика: сборник материалов Международной конференции студентов, аспирантов и молодых ученых. Красноярск: СФУ, 2016, 1, 73-76 [Mozhny E.G., Stepanov A.G. Reactive power compensation problems. Electric power industry and electromechanics: International Student Conference Proceedings, Krasnoyarsk, SibFU, 2016, 1, $73-76$ (in Russian)]

[11] Скакунов Д.А., Дементьев В.В., Карташев Ю.А., Кузьмин Р.С., Меньшиков В.А., Ящук К.П. Естественные средства компенсации реактивной мощности в системах электроснаб- 
жения $6 . .10$ кВ горнометаллургических предприятий. Горное оборудование и электромеханика, 2009, 2, 19-22 [Skakunov D.A., Dementiev V.V., Kartashev Y.A., Kuzmin R.S., Menshikov V.A., Yashchuk K.P. The Natural Means of Compensating for the Reactive Power in the Systems of Supplying Mining and Metallurgical Enterprises with the $6 \ldots 10 \mathrm{kV}$ Electric Power. Mining equipment and electrical engineering, 2009, 2, 19-22 (in Russian)] 